La Revue

des Droits

de l'Homme

\section{La Revue des droits de l'homme}

Revue du Centre de recherches et d'études sur les droits fondamentaux

$3 \mid 2013$

Revue des droits de l'homme $-\mathrm{N}^{\circ} 3$

\title{
Le renouveau constitutionnel en Égypte et la démocratie
}

Les apports de la constitution du 26 décembre 2012

\section{Malik Boumédiene}

\section{(2) OpenEdition}

Journals

Édition électronique

URL : http://journals.openedition.org/revdh/422

DOI : $10.4000 /$ revdh.422

ISSN : 2264-119X

Éditeur

Centre de recherches et d'études sur les droits fondamentaux

Référence électronique

Malik Boumédiene, "Le renouveau constitutionnel en Égypte et la démocratie », La Revue des droits de I'homme [En ligne], 3 | 2013, mis en ligne le 26 décembre 2013, consulté le 08 juillet 2020. URL : http:// journals.openedition.org/revdh/422 ; DOI : https://doi.org/10.4000/revdh.422

Ce document a été généré automatiquement le 8 juillet 2020

Tous droits réservés 


\title{
Le renouveau constitutionnel en Égypte et la démocratie
}

Les apports de la constitution du 26 décembre 2012

\author{
Malik Boumédiene
}

1 La Tunisie a ouvert la voie au "printemps arabe » qui a pu s'étendre à divers pays comme le Maroc, la Syrie, la Libye ou encore le Bahrein. Quels que soient les pays, le peuple demandait davantage de liberté, la fin des régimes autoritaires, la mise en place d'une véritable démocratie et des conditions de vie plus décentes. C'est ainsi que, concernant l'Égypte, plusieurs éléments étaient présents, fondement à un soulèvement. L'Égypte ${ }^{1}$ est le $3^{\mathrm{e}}$ pays d'Afrique le plus touché notamment par la corruption. Cette corruption a couté, à l'Égypte, entre 2000 et 2008 plus de 6 milliards de dollars par an. En outre, $46 \%$ de la population vit dans la pauvreté. Enfin, l'Égypte apparaissait comme un pays où le pouvoir était exercé de manière autoritaire par le Président Hosni Moubarak en poste depuis 1981. Ainsi, l'Égypte n'a pas échappé à ces mouvements populaires dénommés également "révolutions arabes ». Le 25 janvier 2011 devait être célébrée en Égypte la journée des forces de l'ordre, ce fut le jour choisi par des mouvements de protestation pour manifester en exigeant des réformes. Les manifestations se poursuivent ainsi durant plusieurs jours avec pour but le départ du président Moubarak. Celui-ci, le 11 février, quittera le pouvoir qu'il cédera à un Conseil suprême des forces armées de vingt membres, présidé par le maréchal Mohamed Hussein Tantawi, commandant en chef des forces armées qui par une déclaration le 17 juin s'attribue le pouvoir législatif. À cette même date le Président Morsi est élu et le 12 août, il abroge la décision du Conseil suprême des forces armées du 17 juin tout en s'attribuant le pouvoir législatif. Un projet de Constitution est alors approuvé par l'Assemblée constituante (dont les membres ont été élus par le Parlement c'est-à-dire l'assemblée du Peuple et le Conseil consultatif) le 30 novembre 2012. Il est soumis au référendum le 15 et le 22 décembre et approuvé par $63 \%$ des votants. La nouvelle Constitution est signée le 26 décembre 2012 par le Président Morsi. La nouvelle loi fondamentale se veut apporter un renouveau constitutionnel mettant fin au texte de 1971 et consacrant une nouvelle organisation des pouvoirs constitutionnels. Afin de mettre en évidence les apports de la Constitution du 22 décembre 2012, on peut dire 
que le renouveau constitutionnel est venu consacrer l'idée démocratique en Égypte (I). Néanmoins, cette consolidation démocratique reste fragile (II) comme en témoignent plusieurs éléments.

\section{Le renouveau constitutionnel comme fondement à la consolidation de la démocratie}

2 La démocratie se voit donner une nouvelle impulsion avec la mise en place de la nouvelle Constitution. Ce renouveau démocratique se manifeste à travers d'une part, une consolidation des droits fondamentaux (A) et, d'autre part, la recherche davantage d'équilibre entre les différents pouvoirs (B) limitant ainsi les risques de domination d'un pouvoir sur un autre.

\section{A. Des droits fondamentaux renforcés}

3 La reconnaissance, comme la protection, des droits fondamentaux au sein d'un État constitue aujourd'hui un élément essentiel du caractère démocratique ou pas d'un régime politique. En ce qui concerne l'Égypte, on constate que le nouveau texte fondamental du 22 décembre 2012 reconnait un nombre de droits fondamentaux nouveaux ou, vient en préciser, renforcer d'autres déjà existant. Cette nouvelle orientation constitutionnelle doit semble-t-il être intégrée dans un mouvement d'ensemble qui a touché plusieurs pays arabes ces dernières années (Par exemple le Maroc avec la réforme constitutionnelle en date de 2011). C'est ainsi que le droit de propriété bénéficie d'une attention particulière puisqu'il est non seulement garantie mais également "protégé» (article 21 de la Constitution). La liberté personnelle devient un droit protégé et «inviolable». À ce titre, le droit à la sureté est consolidé dans la mesure où il est reconnu le droit de toute personne arrêtée ou détenue d'être tenue informée des motifs de son arrestation (article 35 de la Constitution). Elle ne peut, en outre, être interrogée qu'en présence d'un avocat qui doit être désigné en cas de nécessité. De plus, toute personne arrêtée ou détenue a le droit d'exercer un recours devant un tribunal qui devra se prononcer dans un délai d'une semaine. Dans le cas contraire, elle doit être remise immédiatement en liberté. Le nouveau texte reprend l'idée de l'inviolabilité du domicile et vient encadrer les atteintes éventuelles (article 39). En effet, il est permis d'entrer au domicile d'une personne, de le perquisitionner, ou de le surveiller que dans des conditions strictement déterminées par la loi et sur ordonnance judiciaire motivée, précisant le lieu, la date et l'objectif de la perquisition. En outre, les habitants de la maison doivent être prévenus avant toute perquisitionner. La liberté de culte va bénéficier d'une nouvelle portée puisque, dorénavant, il est indiqué, explicitement, qu'une telle liberté comprend également la construction de lieu de culte. Le constituant semble avoir tenu compte de la composition religieuse du pays et permettre à chaque culte (dont notamment la minorité Chrétienne) de s'épanouir. Au sein des régimes autoritaires, on peut constater que la liberté de la presse est généralement restreinte. Il en a été ainsi en Libye, il en est ainsi en Syrie et au Maroc actuellement (le Maroc ${ }^{2}$ est placé à la $136^{e}$ place sur 179 pays en ce qui concerne la liberté de la presse). Rares sont les articles de presse critiquant la monarchie au Maroc par exemple. La nouvelle Constitution égyptienne, elle, se veut libérale et protectrice. Il est mis l'accent sur le fait que la presse agit de manière 
indépendante dans le but de servir la société, elle a pour objectif d'exprimer les tendances de l'opinion générale et contribue à la formation de celle-ci. La liberté de réunion, qui est fondamentale au sein d'une démocratie, est davantage protégée par rapport au texte de 1971 puisqu'il est précisé qu'il est, non seulement, interdit aux agents de sécurité de participer à ses réunions mais également il est interdit de mettre sur écoute celles-ci (article 50). Il semble qu'il s'agit ici de rompre avec les pratiques antérieures témoignant d'un véritable régime policier. Les libertés collectives du travail n'échappent pas à ce mouvement libéral. Il s'agit pour le constituant de reconnaitre la place des syndicats dans la sphère sociale. Cette nouvelle approche en direction des syndicats se traduira par le fait que la dissolution de ces derniers ne peut dorénavant intervenir que sur décision de justice écartant l'intervention discrétionnaire du pouvoir exécutif.

Les droits sociaux ne resteront pas à l'écart de ce mouvement de consolidation des droits fondamentaux. En effet, il semble que le constituant ait pris toute la mesure de l'importance de ces droits. Il s'agit ainsi de répondre au cadre international également puisque de tels droits sont reconnus notamment par la Déclaration Universelle des Droits de l'Homme de 1948 ou encore par le Pacte international relatif aux droits économiques, sociaux et culturels en date du 16 décembre 1966. Le nouveau texte fondamental dispose ainsi que l'État doit assurer les services de l'assurance sociale, tout citoyen a le droit à la solidarité sociale, s'il est incapable de prendre en charge sa propre personne, ni sa famille, ou en cas d'incapacité, de chômage, de vieillesse, afin de leur assurer un minimum de moyen d'existence (article 66). Les nouvelles dispositions viennent mettre à la charge de l'État l'obligation d'assurer une pension convenable aux petits paysans, aux ouvriers agricoles, à la main d'œuvre saisonnière et à tous ceux qui ne sont pas couvert par le système de l'assurance sociale. La référence au droit au logement apparait aussi comme une nouveauté (article 68). Dans ce sens le droit à une habitation convenable est reconnu. À cette fin l'état a obligation d'adopter un plan national pour le logement basé sur la justice sociale, et qui encourage les initiatives individuelles et les coopératives de l'habitat. Cette reconnaissance du droit à un logement décent répond à une situation dramatique du logement en Égypte puisque plus de 12 millions d'habitants vivent dans des bidonvilles ${ }^{3}$. Cette intégration des droits sociaux au sein des droits fondamentaux témoigne d'une idée essentielle consistant à dire que la liberté n'a aucun sens si les citoyens ne peuvent accéder aux droits élémentaires leur garantissant un minimum de dignité. Enfin, on remarque que la protection de l'enfance fait l'objet d'une attention particulière, contrairement au texte de 1971, allant dans le sens du respect des standards internationaux et de la philosophie de la Convention internationale relative aux droits de l'enfant datant de 1989. Il est fait ainsi interdiction d'embaucher un enfant avant l'âge de la fin de l'enseignement obligatoire (en Égypte, les enfants seraient 7 millions à travailler), dans des activités inappropriées à son âge, ou qui l'empêche de poursuivre ses études. En matière judiciaire, l'enfant ne peut être détenu que pour une durée déterminée et une assistance juridique doit lui être assurée. Le lieu de détention doit être convenable mettant en place une séparation entre les sexes, l'âge, la nature du crime et l'éloignement des lieux de détention des adultes. Enfin, de manière générale, l'article 71 de la loi fondamental souligne que l'État doit prendre soin des enfants et des jeunes, assurer leur éducation, leur développement spirituel, moral, culturel, scientifique, physique, psychologique, social et économique tout en œuvrant pour leur participation politique. 


\section{B. Un équilibre des pouvoirs consacré}

Nous pouvons remarquer que le texte fondamental tend à vouloir modifier le jeu entre les différents pouvoirs constitutionnels, et cela, dans le sens de plus d'équilibre. Une telle volonté se manifeste, semble-t-il, à travers l'affaiblissement de la fonction présidentielle (1) tout en préservant le statut du pouvoir législatif qui prévalait antérieurement dans le texte de 1971 (2).

\section{L'affaiblissement présidentiel}

6 Une première remarque s'impose ici. Il est important de souligner que la réduction des prérogatives du pouvoir exécutif semble apparaître comme une tendance touchant le nouveau constitutionnalisme arabe dans son ensemble suite au "printemps arabe" (voir, par exemple, la Constitution Syrienne en date de 2012). En Égypte, la réduction des prérogatives présidentielles se matérialise de différentes façons. Encadrement des pouvoirs du Chef de l'État, dans un premier temps, comme en témoignent, par exemple, les dispositions relatives au droit de dissolution (article 127). En effet dorénavant le Président de la République ne peut dissoudre la Chambre des députés qu'après un référendum et sur la base d'un décret motivé. En outre, on remarque que le mandat du chef de l'État se voit réduit passant de 6 à 4 ans et, celui-ci ne peut exercer que deux mandats successifs. La déclaration de l'État d'urgence par le chef de l'État est davantage encadrée. En effet, alors que sous le régime de la Constitution de 1971 cette déclaration devait être soumise dans les 15 jours à l'assemblée législative ce délai est ramené à 7 jours. Cet encadrement de l'état d'urgence s'exprime également par le fait que si la déclaration est faite dans une période d'intersession, il faut alors convoquer la Chambre immédiatement pour lui soumettre la déclaration. De même, la loi fondamentale souligne qu'en cas de dissolution de la chambre la déclaration de l'état d'urgence est soumise au Conseil consultatif. Enfin, il est souligné que la majorité des membres des deux chambres doit approuver l'état d'urgence qui doit être établi pour une durée déterminée et ne doit pas excéder 6 mois (un tel délai ne figurait pas dans le texte de 1971).

7 L'affaiblissement de la fonction présidentielle s'exprime non seulement par un plus grand encadrement des prérogatives du chef de l'État mais aussi par la suppression de différentes prérogatives. On observe, par exemple, que la Constitution de 2012 vient supprimer les dispositions telles que celles contenues au sein de l'article 74 de la Constitution de 1971 et qui concernaient les pouvoirs exceptionnels du chef de l'État. Cet article disposait, en effet, que : "En cas de danger imminent et grave menaçant l'unité national, ou la sécurité de la patrie, ou empêchant les institutions de l'État de remplir leur rôle constitutionnel, il appartient au président de la République de prendre les mesures urgentes pour parer à ce danger après consultation du Premier ministre et des présidents de l'assemblée du Peuple et de l'Assemblée consultative. Il adresse un message au peuple et fait procéder à un référendum sur les mesures prises dans les 60 jours qui suivent. La dissolution de l'Assemblée du peuple et de l'Assemblée consultative est interdite lors de l'exercice de ces pouvoirs ». 


\section{Le pouvoir législatif : un statut maintenu}

8 Il semble que la Constitution de 2012 n'apporte pas de changements significatifs quant aux prérogatives du pouvoir législatif. Néanmoins, on peut constater qu'elle maintient, dans ces grandes lignes, sans l'affaiblir, le régime juridique existant sous la Constitution de 1971.

9 Dans un premier temps on observe ainsi que si le chef de l'État possède un certain nombre de prérogatives nous avons une intervention du pouvoir législatif. On remarque dans ce sens que s'il revient au Président de déclarer la guerre c'est après approbation de la Chambre des députés à la majorité de ses membres. De même, si le chef de l'État a la possibilité de mettre son veto à la promulgation d'une loi ce veto n'est pas absolu puisque si la Chambre des députés, lors de la deuxième discussion, vote le texte à la majorité des deux tiers de ses membres le Président de la République doit en assurer la promulgation. La déclaration de l'état d'urgence relève du Chef de l'État mais celle-ci doit être soumise à la Chambre des députés dans les 7 jours suivants.

Le pouvoir législatif possède des moyens de pression sur le pouvoir exécutif. La Chambre des députés a la possibilité de remettre en cause la responsabilité du pouvoir exécutif. En effet, la Chambre des députés peu retirer sa confiance au Premier ministre, à un vice Premier ministre ou à un ministre. Une telle motion de censure ne peut être présentée qu'après interpellation et sur un dixième des députés et doit alors être approuvée par un vote à la majorité des membres composant la Chambre. Dans le cas où la motion de censure concerne le premier ministre ou un ministre et que le gouvernement se solidarise avec lui avant le vote, le gouvernement doit alors démissionner. Les moyens de pression se matérialisent également par le fait que la Chambre peut créer une commission ad hoc ou charger une de ses commissions d'examiner l'activité d'une administration, institution ou entreprise publique, pour s'enquérir sur une affaire et informer la Chambre sur sa situation financière, administrative ou économique, et mener des enquêtes concernant des activités passées. Afin d'accomplir sa mission le texte fondamental précise que la commission peut rechercher des preuves, demander l'audition de ceux qu'elle souhaite interroger. Toutes les autorités doivent accéder à ses demandes et mettre à sa disposition les documents qu'elle juge utile. Dans le sens du contrôle gouvernemental, nous pouvons citer l'article 106 de la Constitution qui permet à 20 députés au moins de la Chambre ou dix du Conseil consultatif de demander l'ouverture d'un débat sur une question générale pour obtenir des éclaircissements sur la politique gouvernementale à son sujet. De manière générale, on observe que tout membre de la Chambre a le droit d'adresser une interpellation au Premier ministre, à un vice premier ministre ou à un ministre pour leur demander des comptes sur une affaire entrant dans leurs attributions.

11 Il apparaît au vu de ces quelques développements que la loi fondamentale de décembre 2012 vient consolider incontestablement la démocratie Egyptienne (du moins dans le texte constitutionnel!). Néanmoins une telle avancée trouve différentes limites qu'il appartiendra de combler dans l'avenir sous peine de l'affaiblir. 


\section{La consolidation démocratique à l'épreuve du renouveau constitutionnel}

12 La démocratie suppose tant une protection aussi large que possible des droits fondamentaux que l'existence d'un équilibre des pouvoirs. Dans le cas de l'Égypte, il semble que ces deux éléments, s'ils sont présents, méritent d'être relativisés. À ce titre, il apparait nécessaire d'aller plus loin tant dans la protection des droits fondamentaux (A) que dans l'équilibre des pouvoirs (B).

\section{A. Les limites de la protection des droits fondamentaux}

Il semble que l'Égypte ne soit pas allée assez loin dans la reconnaissance et la protection des droits et libertés fondamentales entrainant un certain nombre de critiques. De manière générale, dans un premier temps, on peut remarquer que le texte fondamental ne fait aucunement référence au droit international des droits de l'homme. La Constitution aurait pu mettre l'accent sur l'attachement aux droits de l'homme tels que reconnus par la Déclaration Universelle des Droits de l'Homme adoptée dans le cadre des nations unies en 1948. À titre de contre-exemple, nous pouvons citer la Constitution marocaine qui fait référence implicitement au droit international au sein de son préambule qui précise que le Maroc "réaffirme son attachement aux droits de l'Homme tels qu'ils sont universellement reconnus ». En outre, pour compléter cette première remarque d'ordre général, on peut constater que la Constitution ne donne aucune définition de la notion de liberté contrairement au texte fondamental français qui dispose, au sein de l'article 4 de la déclaration des droits de l'homme et du citoyen, que "La liberté consiste à pouvoir faire tout ce qui ne nuit pas à autrui».

On constate également l'absence de protection pour plusieurs sujets. Par exemple, aucune disposition ne concerne l'égalité entre hommes et femmes et la question des discriminations sur la base du sexe contrairement à la Constitution du Bahreïn qui dispose dans son article 18 que : "Il n'y a aucune discrimination entre eux sur la base du sexe ». Il aurait été opportun de préciser que la loi protège et garantie l'égalité entre hommes et femmes. La Constitution du Royaume du Maroc dispose, dans ce sens, que "L'homme et la femme jouissent, à égalité, des droits et libertés à caractère civil, politique, économique, social, culturel et environnemental, énoncés dans le présent titre et dans les autres dispositions de la Constitution, ainsi que dans les conventions et pactes internationaux dûment ratifiés par le Royaume ». Nous pouvons nous inquiéter de la position, sur le statut de la femme, de la confrérie des frères musulmans à laquelle appartient le Président Morsi. En effet cette confrérie, dans un communiqué de mars 2013, s'est opposée à l'octroi aux filles d'une liberté sexuelle totale ou à la mise en place d'une égalité totale en ce qui concerne le statut du mariage. On constate aussi qu'il existe en Égypte une inégalité réelle entre hommes et femmes puisque, par exemple, le taux d'analphabétisme est de $25,4 \%$ pour les hommes et de $42,2 \%$ pour les femmes. De même, on remarque que

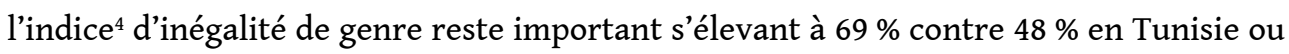
en Libye.

D'autre part, si, comme on l'a vu, des articles reconnaissent certains droits une telle reconnaissance reste timide. Par exemple, il existe des dispositions concernant le droit 
de l'environnement. Cependant, celles-ci ne font aucune référence aux principes de précaution, de participation ou encore de responsabilité environnementale. Concernant le procès pénal plusieurs grands principes sont reconnus comme la présomption d'innocence ou le double degré de juridiction. Néanmoins on peut regretter l'absence de référence à la notion de droit à réparation ou encore du droit de voir sa cause entendu et cela dans un délai raisonnable. Egalement, est reconnue la liberté de croyance, mais il aurait nécessaire de mentionner que nul ne peut être inquiété pour ses opinions religieuses d"autant que la religion musulmane est religion d'État (article 2 de la Constitution). Cette protection de la liberté religieuse est d'autant plus relative qu'il semble que nous soyons en présence d'une domination de l'islam. C'est ainsi que la conversion au christianisme est rarement tolérée et fait l'objet d'une procédure spécifique. On peut observer que les personnes souhaitant se convertir au christianisme peuvent faire l'objet d'une répression de la part des pouvoirs publics. Les coptes sont soumis à un régime dérogatoire en ce qui concerne la possibilité d'exercer leur culte. Pour construire une église il est nécessaire d'obtenir une autorisation du Chef de l'État. Il est fait également interdiction de construire une église à proximité d'une mosquée. En outre, afin de construire une église il faut justifier de la présence d'une communauté chrétienne à proximité. Cette domination de l'islam s'exprime au sein de la législation sur les mariages mixtes puisque la loi souligne que dans les mariages mixtes la religion la «meilleure » s'impose, c'est-à-dire l'islam.

La liberté des médias et de manière générale la liberté de la presse peut faire l'objet d'une censure dans des situations particulières comme par exemple en temps de guerre ou de mobilisation générale (article 48). Or la loi constitutionnelle ne vient aucunement préciser ce qu'il faut entendre par «mobilisation générale». Cette absence de précision peut donner lieu à des interprétations pouvant entrainer des abus.

Les grandes libertés fondamentales doivent être étudiées en les confrontant à la situation politique du moment. À ce titre, s'agissant de la liberté d'expression en Égypte, malgré une reconnaissance constitutionnelle la réalité est fort différente. C'est ainsi que début 2013 l'humoriste Youssef Bassem, bénéficiant d'une notoriété en Égypte, a fait l'objet de plusieurs plaintes, accusé d'avoir insulté le Président et la religion musulmane ${ }^{5}$. Gamal EID, qui est avocat et Directeur du réseau arabe d'information des droits de l'homme, a déclaré récemment que depuis l'investiture du Président Morsi, on observe quatre fois plus de poursuites pour insultes au Président.

19 Une question fondamentale se pose également. Les droits et libertés reconnus par la loi fondamentale restent conditionnés par leur mise en œuvre par le législateur. Il y a ainsi dans un premier temps, la nécessité d'une volonté politique. D'autre part, la mise en œuvre de droits tels que le droit au logement, le droit à la santé ou le droit à l'éducation sont des droits qui ont un coût financier important. Quid de la capacité financière de l'Égypte qui apparait comme un pays pauvre? Le PIB se situant au environ de 188 milliards de dollars.

Enfin, concernant la protection des droits fondamentaux le texte constitutionnel met en place un contrôle a priori de la loi. Or, il aurait, là également, opportun d'aller plus loin en donnant la possibilité au justiciable de saisir la Cour constitutionnelle au cours d'un procès. C'est-à-dire la reconnaissance d'un contrôle a posteriori de la loi. À titre de comparaison, un tel contrôle existe, notamment, au sein de la Constitution du Tchad en date du 14 avril 1996 qui dispose, dans son article 166, que «Tout citoyen peut soulever l'exception d'inconstitutionnalité devant une juridiction dans une affaire qui la concerne. Dans 
ce cas, la juridiction sursoit à statuer et saisit le Conseil constitutionnel qui doit prendre une décision dans un délai de quarante-cinq jours ».

\section{B. La fragilité de l'équilibre des pouvoirs}

21 Cette fragilité de l'équilibre des pouvoirs est caractérisée par l'omniprésence présidentielle (1) ainsi que par une certaine mise sous contrôle du pouvoir législatif (2).

\section{L'omniprésence présidentielle}

22 On observe que le Président de la République reste omniprésent au sein de la Constitution de décembre 2012 à l'instar de la Constitution Syrienne ou du Royaume du Maroc (prépondérance royale). Plusieurs dispositions tendent à appuyer une telle idée. Il lui revient, par exemple, de convoquer les deux chambres en session annuelle et il clos cette session. Le Président de la République possède une pleine place dans la procédure législative puisqu'il a la possibilité de proposer des lois. En outre, il a le droit d'opposer son veto à la promulgation d'une loi et demander, à ce titre, à la Chambre des députés d'en débattre une nouvelle fois. Le chef de l'État nomme un certain nombre de membres du Conseil consultatif. L'article 132 de la nouvelle Constitution précise qu'il est le chef de l'exécutif. De manière générale, il lui appartient de veiller aux intérêts du peuple, de sauvegarder l'indépendance de la nation et l'intégrité du territoire et il doit garantir la séparation des pouvoirs. Il nomme le Premier ministre et "le charge » de former son Gouvernement. Le texte fondamental donne un rôle important au chef de l'État dans la détermination de la politique nationale puisqu'il lui revient d'élaborer la politique générale avec «la participation » du Conseil des ministres. Il a la possibilité de convoquer le Gouvernement à chaque fois qu'il le juge utile. En effet, l'article 143 dispose que "Le président de la République a le droit de convoquer le gouvernement à une réunion de concertation pour examiner les question importantes, de présider la réunion et de demander, au Premier ministre, tous les rapports qu'il souhaite sur les affaires publiques ». Le chef de l'État apparait comme le chef des armées. À ce titre c'est à lui qu'il revient de déclarer la guerre. Il a la prérogative de déclarer l'état d'urgence également. Une prérogative importante appartient au chef de l'État puisqu'il a la possibilité de saisir le peuple par voie de referendum sur toute question concernant les intérêts supérieurs de l'État. Un tel referendum, précise la Constitution, "est contraignant " pour toutes les autorités et pour l'ensemble de la population. Un tel référendum lui permet ainsi d'imposer ses points de vue sur des questions diverses. Le Président semble posséder également une influence au niveau du pouvoir judiciaire ce qui nous conduit à nous interroger sur la question de la séparation des pouvoirs et l'indépendance du pouvoir judiciaire. En effet, la Constitution précise que le parquet est dirigé par un Procureur général nommé par décret du Président de la République sur proposition du Conseil supérieur de la magistrature. Le procureur général est choisi parmi les vice-présidents de la Cour de cassation, les présidents des cours d'appel et les procureurs généraux adjoints. Enfin, le chef de l'État tient une place dans la procédure de révision constitutionnelle dans la mesure où il a le droit de proposer un ou des amendements aux articles de la Constitution (article 217).

Cette omniprésence du Chef de l'État se matérialise aussi par son intervention indirecte dans le domaine législatif. En effet, la Constitution prévoit qu'en cas de dissolution de la Chambre des députés, il revient au Conseil consultatif d'exercer le pouvoir législatif. Or, 
il faut savoir qu'une partie des membres de ce Conseil est nommée par le Président de la République. Ce dernier possèdera ainsi de relais puissants afin d'imposer sa politique tant en situation normale qu'en cas de dissolution.

\section{Un pouvoir législatif sous contrôle gouvernemental}

Nous remarquons que non seulement le chef de l'État intervient dans la procédure législative mais que le pouvoir législatif se trouve sous contrôle du Gouvernement. Nous pouvons rapprocher cette idée de la Constitution française qui attribue une place prépondérante à l'exécutif également. Plusieurs éléments semblent pousser dans ce sens. C'est ainsi que le Gouvernement a la possibilité de convoquer une des deux Chambres à tenir un session extraordinaire. L'article 101 donne compétence au Gouvernement afin de rédiger des propositions de lois. On peut s'interroger sur la capacité pour la Chambre des députés à mettre en cause la responsabilité de l'ensemble du Gouvernement. En effet, en cas de dépôt et de vote d'une motion de censure le Gouvernement doit démissionner à partir du seul moment où il se solidarise avec le Premier ministre ou un ministre visé par une motion de censure. L'article 161 permet à chaque ministre de prononcer une déclaration devant une des deux chambres ou devant une de leur commission sur une question qui rentre dans leurs prérogatives. Une telle possibilité permet à l'exécutif d'encourager l'adoption de lois dans le sens souhaité. En outre, aucune disposition ne vient concerner les modalités de détermination de l'ordre du jour de la Chambre des députés. On peut en déduire qu'il appartient à cette dernière de fixer l'ordre du jour. Néanmoins, l'expérience peut nous amener à affirmer que le Premier Ministre, chef de la majorité, semble posséder une marge de manœuvre importante afin d'imposer ses choix en ce qui concerne la priorité des propositions de lois devant être débattues en priorité.

\section{Conclusion}

La Constitution du 26 décembre 2012 apparaît, incontestablement, comme une avancée démocratique et va dans le sens du mouvement libéral du renouveau constitutionnel arabe suite au «Printemps arabe». Cependant, l'architecture constitutionnelle mériterait d'être améliorée afin de donner toute sa portée au concept de démocratie. En outre, il ne faut pas écarter la question de la pratique du pouvoir qui participera également à définir la réalité du régime politique. Les constituants ont souhaité, semble-t-il, mettre en place un régime parlementaire, consacrer un équilibre des pouvoirs. Or, sur la base du texte fondamental il apparaît que l'on peut se retrouver très vite en présence d'un exécutif tout puissant reléguant le Parlement à une simple chambre d'enregistrement. Une autre question se pose également. Il s'agit de la place consacrée à l'opposition puisque l'on constate qu'il n'y a pas de protection constitutionnelle du statut de l'opposition contrairement au Maroc par exemple. De même, on peut s'interroger sur l'indépendance de la fonction juridictionnelle qui apparait comme un élément constitutif de l'État de droit. En effet, on peut remarquer que l'article 173 de la Constitution dispose que "Le parquet est dirigé par un procureur général nommé par décret du président de la République ». Enfin, la démocratie, il l'a souvent été souligné, suppose une éducation et une information. Pierre PACTET ${ }^{6}$, précise qu'un choix "ne peut être éclairé que s'il est le fait d'un citoyen conscient, apte à confronter les programmes et les raisonnements... ». Concernant, l'Égypte on peut constater que le taux 
d'analphabétisme demeure important, d'où l'interrogation sur la capacité de choix des citoyens égyptiens !

\section{NOTES}

1. Organisation Global Financial Integrity, rapport 2011.

2. RSF, rapport, Classement mondial de la liberté de la presse, 2013

3. Amnesty international, communiqué de presse, 23 août 2011 http://www.amnesty.org/fr/for-media/ press-releases/egypt-stop-forced-evictions-and-consult-slum-dwellers-resolve-housing-crisi

4. Il s'agit d'un indicateur des inégalités de genre établi par l'ONU. Cet indice est une mesure composite qui reflète les inégalités des réalisations entre hommes et femmes dans trois dimensions : la santé productive ; l'autonomisation et le marché du travail. Plus le pourcentage est élevé plus les inégalités sont grandes

5. L'article 44 de la Constitution dispose que tout dénigrement ou diffamation de l'ensemble des messagers et des prophètes est interdit.

6. Droit constitutionnel, Pierre PACTET, Ferdinand MEIN-SOUCRAMANIEN, Sirey Université, 2009.

\section{AUTEUR}

\section{MALIK BOUMÉDIENE}

Maître de conférences (HDR), Université Toulouse 2 